\title{
Covariance analysis on the thermal neutron capture cross sections using an Am-Be neutron source
}

\author{
Priyada Panikkath ${ }^{1,2, *}$, Naohiko Otuka ${ }^{3,4}$, and P. Mohanakrishnan ${ }^{1}$ \\ ${ }^{1}$ Manipal Centre for Natural Sciences, Manipal Academy of Higher Education, Manipal 576104, India \\ ${ }^{2}$ Department of Physics, Alagappa University,Karaikudi 630004, India \\ ${ }^{3}$ Nuclear Data Section, Division of Physical and Chemical Sciences, Department of Nuclear Sciences and Applications, International \\ Atomic Energy Agency, A-1400 Wien, Austria \\ ${ }^{4}$ Nishina Center for Accelerator-Based Science, RIKEN, Wako, Saitama 351-0198, Japan
}

\begin{abstract}
We measured the thermal cross sections of ${ }^{71} \mathrm{Ga}(\mathrm{n}, \gamma)^{72} \mathrm{Ga}$ using an Am-Be neutron source having strength of $4 \times 10^{7}$ neutrons/sec using the two monitor reactions ${ }^{197} \mathrm{Au}(\mathrm{n}, \gamma){ }^{198} \mathrm{Au}$ and ${ }^{55} \mathrm{Mn}(\mathrm{n}, \gamma){ }^{56} \mathrm{Mn}$, and obtained $4.07 \pm 0.27 \mathrm{~b}$ and $3.97 \pm 0.31 \mathrm{~b}$ as the values measured relative to $\mathrm{Au}$ and $\mathrm{Mn}$ neutron captures, respectively. We constructed their covariance matrix by taking into account the correlation of parameters which are not independent in derivation of these two thermal cross sections (e.g., Ga sample weight, decay data of ${ }^{72} \mathrm{Ga}$, detector efficiency of $\gamma$-ray emitted from ${ }^{72} \mathrm{Ga}$ ). The off-diagonal weighted mean of the two cross sections $4.04 \pm 0.27 \mathrm{~b}$ was derived from the two thermal cross sections and their covariance matrix.
\end{abstract}

\section{Introduction}

There have been a number of publications reporting the ${ }^{71} \mathrm{Ga}(\mathrm{n}, \gamma){ }^{72} \mathrm{Ga}$ thermal cross section [1-13] according to the EXFOR library [14]. Most of these experimental studies used reactor neutrons for irradiation. The thermal neutron cross section measured with mono energetic cold neutrons for irradiation [7] is considerably lower than the majority of the measured cross sections in the literature. Under this situation, we measured the ${ }^{71} \mathrm{Ga}(\mathrm{n}, \gamma){ }^{72} \mathrm{Ga}$ thermal cross section using an Am-Be neutron source relative to the two monitor reactions ${ }^{197} \mathrm{Au}(\mathrm{n}, \gamma){ }^{198} \mathrm{Au}$ and ${ }^{55} \mathrm{Mn}(\mathrm{n}, \gamma){ }^{56} \mathrm{Mn}$. We obtained the covariance between the two thermal cross sections measured with the two monitor reactions, and applied it for derivation of the off-diagonal weighted mean [15] of these thermal cross sections.

\section{Experimental}

Our experiment was carried out in the Am-Be neutron source facility at Manipal Centre for Natural Sciences (MCNS), Manipal Academy of Higher Education. The neutron source was kept inside a concrete bunker and thus the neutrons got moderated. The neutron spectrum has a mixed component including thermal and epithermal neutrons. Details of the neutron source facility and the spectrum are available in our previous publications [16-18]. Analytical grade $\mathrm{Ga}_{2} \mathrm{O}_{3}$ powder of Alfa Aesar with purity of $99.995 \%$ was prepared in small packets. Mn and $\mathrm{Au}$ foils (12.7 $\mathrm{mm}$ in diameter) procured from Shieldwerx with purity $99.9 \%$ were used as the monitor foils. Two

*e-mail: priyadapv@gmail.com sets of Ga sample and monitor foils were used in the experiment: One of them was enclosed in a standard Cd cover (1.02 mm thick) and the other without the $\mathrm{Cd}$ cover. The Ga sample and Mn monitor foil were irradiated together for 5 days. The Au monitor foils with and without the Cd cover were irradiated separately for 32 days since the half-life of ${ }^{198} \mathrm{Au}$ is longer than those of ${ }^{72} \mathrm{Ga}$ and ${ }^{56} \mathrm{Mn}$. The induced activity in each irradiated material was determined from the corresponding gamma spectra. Gamma spectrum measurements were carried out using a 30\% relative efficiency $\mathrm{HPGe}$ detector pre-calibrated using a ${ }^{152} \mathrm{Eu}$ source. The distance between the irradiated material and detector was $2 \mathrm{~cm}$. The coincidence summing effects of the gamma lines of interest were estimated and corrected using a Monte Carlo simulation code EFFTRAN [19]. Each irradiated material was counted for $2500 \mathrm{~s}-60000 \mathrm{~s}$ depending upon the half-life to achieve satisfactory counting statistics.

\section{Data reduction}

The reaction rate per target atom $(R)$ was estimated from the net area $(C)$ under the full peak of the respective gamma-line by

$$
R=\frac{C T M f_{a} f_{c}}{N_{A} \theta \epsilon I_{\gamma} m}
$$

with

$$
T=\frac{\lambda}{\left(1-e^{-\lambda t_{i}}\right) e^{-\lambda t_{d}}\left(1-e^{-\lambda t_{c}}\right)},
$$

where $M$ is the molar mass, $f_{a}$ is the gamma attenuation factor, $f_{c}$ is the coincidence summing correction factor, $N_{A}$ is the Avogadro constant, $\theta$ is the isotopic abundance, $\epsilon$ is 
Table 1. Decay data (gamma energy $E_{\gamma}$, gamma intensity $I_{\gamma}$, half-life $T_{1 / 2}$ )[20-22], thermal constants (Westcott factor $g$, thermal cross section $\sigma_{0}$ [23]), gamma attenuation factor $f_{a}$, coincidence summing correction factor $f_{c}$, Cd transmission factor $F_{\mathrm{Cd}}$ [24], and thermal self-shielding factor $G_{\text {th }}$ adopted in our work. The value of the uncertainty in parenthesis applies to the least significant digits, i.e., 95.45(8) means $95.45 \pm 0.08$.

\begin{tabular}{cccccccccc}
\hline Product & $E_{\gamma}(\mathrm{keV})$ & $I_{\gamma}(\%)$ & $T_{1 / 2}$ & $g$ & $\sigma_{0}(\mathrm{~b})$ & $f_{a}$ & $f_{c}$ & $F_{\mathrm{Cd}}$ & $G_{\text {th }}$ \\
\hline${ }^{72} \mathrm{Ga}$ & 834.13 & $95.45(8)$ & $14.10(2) \mathrm{h}$ & 1.001 & - & 1.003 & 1.084 & 1.00 & 0.999 \\
${ }^{198} \mathrm{Au}$ & 411.80 & $95.62(6)$ & $2.6941(2) \mathrm{d}$ & 1.006 & $98.65(9)$ & 1.009 & 1.001 & 0.991 & 0.983 \\
${ }^{56} \mathrm{Mn}$ & 846.76 & $98.85(3)$ & $2.5789(1) \mathrm{h}$ & 1.001 & $13.36(5)$ & 1.005 & 1.025 & 1.0 & 0.997 \\
\hline
\end{tabular}

the detector efficiency, $I_{\gamma}$ is the gamma intensity, $m$ is the sample mass, $\lambda$ is the decay constant of the capture product, $t_{i}$ is the irradiation time, $t_{d}$ is the cooling time, and $t_{c}$ is the counting time. The decay data in Eq. (2) were taken from the ENSDF library [20-22], and are listed in Table 1 . The gamma attenuation factor $f_{a}$ was determined using the linear attenuation coefficient $(\mu)$ taken from the XCOM photon cross section database [25], and the coincidence summing correction factor $f_{c}$ was calculated by the EFFTRAN code.

The neutron capture thermal cross sections of the sample $\sigma_{0, S}$ was determined by

$$
\sigma_{0, S}=\frac{\left[R-R_{\mathrm{Cd}} / F_{\mathrm{Cd}}\right]_{S}}{\left[R-R_{\mathrm{Cd}} / F_{\mathrm{Cd}}\right]_{M}} \frac{\left[G_{\mathrm{th}} g\right]_{M}}{\left[G_{\mathrm{th}} g\right]_{S}} \sigma_{0, M},
$$

$[16,17,26]$ where $G_{\text {th }}$ is the thermal self-shielding factor, $g$ is the Westcott factor, and $\sigma_{0, M}$ is the neutron capture thermal cross section of the monitor foil. The self-shielding factor $G_{\text {th }}$ was estimated analytically as in Ref. [27]. The multiple scattering effect was also studied by using the Monte Carlo simulation code PHITs [28], which confirmed that the effect is negligible in our experimental configuration.

\section{Characterization of efficiency curve}

The efficiency of the HPGe detector was calibrated by counting nine major gamma lines of a ${ }^{152} \mathrm{Eu}$ standard reference source and the ${ }^{152} \mathrm{Eu}$ decay data in the ENSDF library [29]. The measured efficiencies for the nine gamma lines were corrected for the coincidence summing effect by using the EFFTRAN code, and fitted by

$$
\ln \epsilon=a_{0}+a_{1} \ln E
$$

by the least-squares method. We have ignored the uncertainties in the ${ }^{152} \mathrm{Eu}$ half-life and calibration source activity since they are commonly applied to the nine gamma lines, while the uncertainties in the counting statistics and gamma intensities were propagated to the uncertainties in $a_{0}$ and $a_{1}$. Since the efficiencies appear in the form of their ratios in Eq. (3), the covariance of the fitting parameters $\operatorname{Cov}\left(a_{i}, a_{j}\right)$ was propagated to the covariance of the efficiency ratios $\operatorname{Cov}\left(\eta_{1}, \eta_{2}\right)$, where $\eta_{1}=\epsilon\left({ }^{198} \mathrm{Au}\right) / \epsilon\left({ }^{72} \mathrm{Ga}\right)$ and $\eta_{2}=\epsilon\left({ }^{56} \mathrm{Mn}\right) / \epsilon\left({ }^{72} \mathrm{Ga}\right)$. The uncertainties and correlation coefficients of the detection efficiency ratios are summarized in Table 2. More detailed explanation and formulation about the uncertainty propagation can be found elsewhere [18].

\section{Uncertainty and covariance analysis}

There have been a number of publications reporting the thermal neutron cross section of ${ }^{71} \mathrm{Ga}(\mathrm{n}, \gamma)^{72} \mathrm{Ga}$ reaction. However, most of the experimental works report only overall uncertainties without any details.

In our work, the detailed analysis of uncertainty and correlation are essential because our goal is to determine the mean of the two thermal cross sections determined with two monitor foils ( $\mathrm{Au}$ and $\mathrm{Mn})$. The fractional $\left(\%^{2}\right)$ covariance between $\sigma_{0 i}$ and $\sigma_{0 j}$ is

$$
\operatorname{cov}\left(\sigma_{0 i}, \sigma_{0 j}\right)=\sum_{k} S_{i k} \frac{\Delta x_{i k}}{x_{i k}} \operatorname{cor}\left(x_{i k}, x_{j k}\right) \frac{\Delta x_{j k}}{x_{j k}} S_{j k},
$$

where

$$
S_{i k}=\frac{x_{i k}}{\sigma_{0 i}} \frac{\partial \sigma_{0 i}}{\partial x_{i k}}
$$

is the (fractional) sensitivity coefficient of $\sigma_{0 i}$ to $x_{i k}$ [30]. Our detailed analysis [18] shows that the sensitivity coefficient $S_{i k}$ often deviates from one, hence the typical quadrature summation in the uncertainty propagation cannot be used.

\section{Results and discussion}

The newly determined ${ }^{71} \mathrm{Ga}$ thermal neutron capture cross sections with the monitor reactions ${ }^{197} \mathrm{Au}(\mathrm{n}, \gamma){ }^{198} \mathrm{Au}$ and ${ }^{55} \mathrm{Mn}(\mathrm{n}, \gamma){ }^{56} \mathrm{Mn}$ are $4.07 \pm 0.27 \mathrm{~b}(6.68 \%)$ and $3.97 \pm 0.31 \mathrm{~b}$ $(7.89 \%)^{1}$, respectively, and they are consistent to each other. The total uncertainty in the thermal cross section is dominated by the uncertainties due to counting statistics of ${ }^{72} \mathrm{Ga}$ for the bare and Cd-covered samples $(5.44 \%$ for the bare sample, $2.67 \%$ for the Cd-covered sample). These partial uncertainties are applicable to the thermal cross sections determined relative to $\mathrm{Au}$ and $\mathrm{Mn}$ monitor reactions since the result of the same $\mathrm{Ga}$ irradiation was shared by both thermal cross sections. Their conventional and off-diagonal weighted means are $4.03 \pm 0.21 \mathrm{~b}$ and $4.04 \pm 0.27 \mathrm{~b}^{2}$, respectively.

The thermal neutron cross section of ${ }^{71} \mathrm{Ga}(\mathrm{n}, \gamma)^{72} \mathrm{Ga}$ reaction determined in our work is shown in Fig. 1 along with other literature values. The uncertainty in our result is slightly higher than many of the earlier measurements. We believe our uncertainty is more realistic because we

\footnotetext{
${ }^{1}$ The uncertainty was revised from $0.32 \mathrm{~b}(8.09 \%)$ [18].As a consequence, the correlation coefficient between the two thermal cross sections was revised to 0.73 from 0.71 [18].

${ }^{2}$ The off-diagonal weighted mean was revised to $4.04 \pm 0.27$ b from $4.05 \pm 0.27 \mathrm{~b}[18]$
} 
Table 2. Efficiency ratios $\eta$ for the $\gamma$ lines used for measurements of the ${ }^{198} \mathrm{Au},{ }^{56} \mathrm{Mn}$ and ${ }^{72} \mathrm{Ga}$ activities [18].

\begin{tabular}{ccccccc}
\hline \hline Product & $\begin{array}{c}\text { Energy } \\
(\mathrm{keV})\end{array}$ & \multicolumn{2}{c}{$\begin{array}{c}\text { Efficiency ratio } \\
\eta\end{array}$} & \multicolumn{2}{c}{$\begin{array}{c}\text { Correlation } \\
\text { coefficient }\end{array}$} \\
\hline${ }^{198} \mathrm{Au} /{ }^{72} \mathrm{Ga}$ & $411.80 / 834.13$ & 1.76480 & \pm & 0.03071 & 1.00 & \\
${ }^{56} \mathrm{Mn} /{ }^{2} \mathrm{Ga}$ & $846.76 / 834.13$ & 0.98798 & \pm & 0.00037 & -1.00 & 1.00 \\
\hline
\end{tabular}

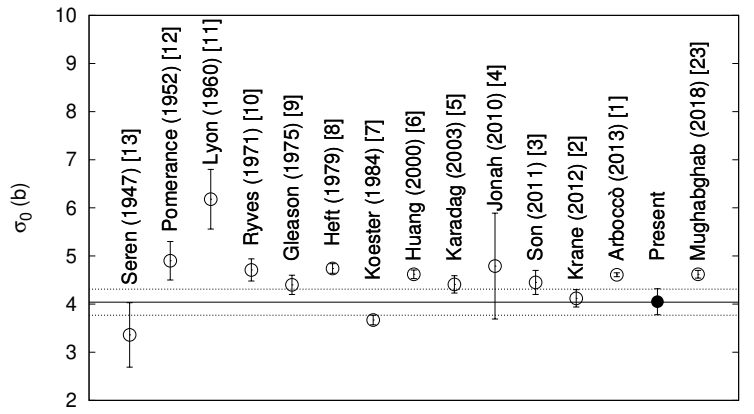

Figure 1. The thermal neutron cross sections of ${ }^{71} \mathrm{Ga}(\mathrm{n}, \gamma)^{72} \mathrm{Ga}$ reaction determined in our measurement (revised from Ref. [18]), other measurements [1-13], and Mughabghab's recommendation [23]. Symbols are sorted by the chronological order on the horizontal axis. The solid and dashed lines show our result and its standard deviation, respectively.

Table 3. Thermal ${ }^{71} \mathrm{Ga}(\mathrm{n}, \gamma)^{72} \mathrm{Ga}$ cross sections from our measurement (revised from Ref. [18]) as well as recommended and evaluated cross sections.

\begin{tabular}{lc}
\hline Our thermal cross sections (b) & \\
\hline Result with ${ }^{197} \mathrm{Au}$ monitor & $4.07 \pm 0.27$ \\
Result with ${ }^{55} \mathrm{Mn}$ monitor & $3.97 \pm 0.31$ \\
Conventional weighted mean & $4.03 \pm 0.21$ \\
Off-diagonal weighted mean & $4.04 \pm 0.27$ \\
\hline \multicolumn{2}{l}{ Recommended and evaluated thermal cross sections (b) } \\
\hline S. Mughabghab (2018) [23] & $4.62 \pm 0.08$ \\
ENDF/B-VII.0 (2018) [31] & 4.73 \\
JEFF-3.3 (2017) [32] & 4.59 \\
BROND-3.1 (2016) [33] & 4.73 \\
CENDL-3.1 (2011) [34] & 4.73 \\
JENDL-4.0 (2011) [35] & 3.71 \\
\hline
\end{tabular}

tried to identify and propagate all major uncertainties in the parameters.

Our off-diagonal weighted mean is lower than the majority of the measurements in the literature, but closer to Koester et al. [7]. Table 3 compares our thermal cross sections with those recommended or evaluated. Our result is lower than recommendation and evaluations in general, but consistent with the JENDL-4.0 [35] evaluation, which adjusts the negative energy level parameters to reproduce Koester's thermal cross section. Among recent measurements, our result also shows good agreement with Krane et al. [2].

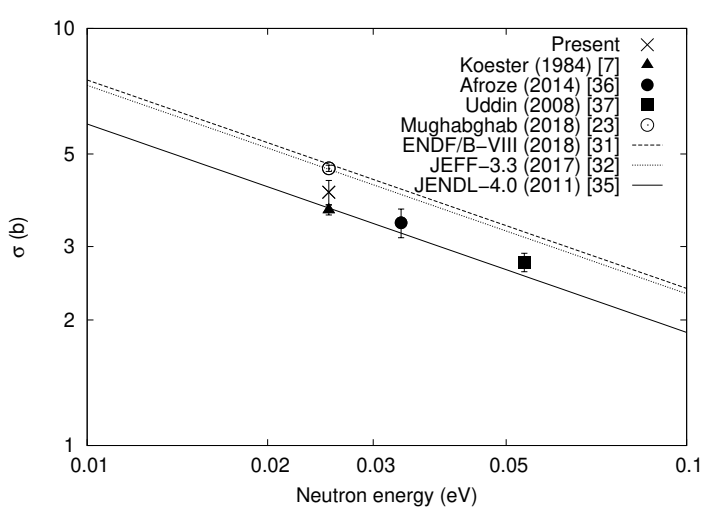

Figure 2. Comparison of our thermal cross section (revised from Ref. [18]) with two cross sections measured with filtered neutron beams at $0.0334 \mathrm{eV}$ [36] and $0.0536 \mathrm{eV}$ [37] and some recommended/evaluated cross sections.

There are two experimental works reporting cross sections measured at energies slightly higher than the thermal energy by using filtered neutrons at $0.0334 \mathrm{eV} \mathrm{[36]} \mathrm{and}$ $0.0536 \mathrm{eV}$ [37]. Figure 2 compares our result with these two experimental cross sections as well as recommended and evaluated thermal cross sections. This figure shows the cross sections reported by us and Koester et al. [7] are consistent with the cross sections measured by Afroze et al. [36] and Uddin et al. [37] assuming the 1/v dependence.

\section{Summary}

We measured the thermal neutron cross section of ${ }^{71} \mathrm{Ga}(\mathrm{n}, \gamma){ }^{72} \mathrm{Ga}$ reaction by irradiating natural Ga samples as well as $\mathrm{Au}$ and $\mathrm{Mn}$ monitor foils with and without a Cd cover by neutrons from an Am-Be source moderated by a concrete bunker. The results obtained with the $\mathrm{Au}$ and Mn monitor foils are consistent, and we adopted their off-diagonal weighted means as our final result by taking into account the covariance between the two results. After publication of the full documentation of our work [18], we reanalyzed the uncertainty propagation, and revised our final thermal cross section value to to $4.04 \pm 0.27 \mathrm{~b}$. The newly obtained thermal neutron cross section is lower than those reported by other recent experiments using neutron fields characterized by broad neutron spectra as well as some evaluated data libraries, but our result compares better with those determined with monoenergetic neutron sources as well as the JENDL-4.0 library. 


\section{Acknowledgments}

We thank to Dr. Yosuke Iwamoto (Japan Atomic Energy Agency) for performing Monte Carlo simulation with the detailed geometry to estimate the fraction of the capture events induced by neutrons scattered from the material using the PHITs code. Dr. Tim Vidmar (SCK.CEN) kindly provided us the EFFTRAN code for coincidence summing corrections. Dr. K. V. Subbaiah (RSO, MCNS) and other staffs of Neutron Physics Laboratory kindly extended their support during the experiments. This work was funded by the Science and Engineering Research Board (SERB), Department of Science and Technology, Government of India under start-up-grant (File No:YSS/2015/000899). It was also partially supported by Manipal Centre for Natural Sciences. PP acknowledges the Post Doctoral Fellowship under RUSA-Phase 2.0.

\section{References}

[1] F. Farina Arboccò, P. Vermaercke, K. Smits, L. Sneyers and K. Strijckmans, J. Radioanal. Nucl. Chem. 296, 931 (2013)

[2] K.S. Krane, Appl. Radiat. Isot. 70, 1649 (2012)

[3] P.N. Son, T.T. Anh, C.D. Vu and V.H. Tan, J. Korean Phys. Soc. 59, 1761 (2011)

[4] S.A. Jonah and U. Sadiq, Report INDC(NDS)-0574, p.67, International Atomic Energy Agency (2010)

[5] M. Karadag, H. Yücel, M. Tan and A. Özmen, Nucl. Instrum. Meth. Phys. Res. A 501, 524 (2003)

[6] Huang Xiao-long, Han Xiao-gang, Yu Wei-xiang, Lu Han-lin and Zhao Wen-rong, Atom. Energy Sci. Technol. 34, 456 (2000)

[7] L. Koester, K. Knopf, W. Waschkowski and A. Klüver, Z. Phys. A 318, 347 (1984)

[8] R.E. Heft, A consistent set of nuclear-parameter values for absolute INAA, in Proceedings of the American Nuclear Society Topical Conference, Mayaguez, Puerto Rico, 1978, p.495

[9] G. Gleason, Radiochem. Radioanal. Lett. 23, 317 (1975)

[10] T.B. Ryves, J. Nucl. Energy 25, 129 (1971)

[11] W.S. Lyon, Nucl. Sci. Eng. 8, 378 (1960)

[12] H. Pomerance, Phys. Rev. 88, 412 (1952)

[13] L. Seren, H.N. Friedlander and S.H. Turkel, Phys. Rev. 72, 888 (1947)

[14] N. Otuka et.al., Nucl. Data Sheets 120, 272 (2014)

[15] W. Mannhart, Report INDC(NDS)-0588, International Atomic Energy Agency (2011)

[16] P. Panikkath and P. Mohanakrishnan, Eur. Phys. J. A 52, 276 (2016)
[17] P. Panikkath and P Mohanakrishnan, Eur. Phys. J. A 53, 46 (2017)

[18] P. Panikkath, N. Otuka, Y. Iwamoto and P. Mohanakrishnan, Eur. Phys. J. A, 55, 91 (2019)

[19] T. Vidmar, Nucl. Instrum. Meth. Phys. Res. A 550, 603 (2005)

[20] D. Abriola and A.A. Sonzogni, Nucl. Data Sheets 111, 1 (2010)

[21] Huang Xiaolong and Kang Mengxiao, Nucl. Data Sheets 133, 221 (2016)

[22] Huo Junde, Huo Su and Yang Dong, Nucl. Data Sheets 112, 1513 (2011)

[23] S.F. Mughabghab, Atlas of Neutron Resonances 6th Edition (Elsevier, Amsterdam, 2018)

[24] T. El Nimr, F. De Corte, L. Moens, A. Simonits and J. Hoste, J. Radioanal. Chem. 67, 421 (1981)

[25] M.J. Berger, J.H. Hubbell, S.M. Seltzer, J. Chang, J.S. Coursey, R. Sukumar, D.S. Zucker and K. Olsen, XCOM: Photon cross sections database, NIST Standard Reference Database 8 (National Institute of Standards and Technology, Gaithersburg, 2010)

[26] H. Yücel, M.G. Budak and M. Karadag, Phys. Rev. C 76, 034610 (2007)

[27] M. Blaauw, Nucl. Instrum. Meth. Phys. Res. A 356, 403 (1995)

[28] T. Sato, Y. Iwamoto, S. Hashimoto, T. Ogawa, T. Furuta, S. Abe, T. Kai, P.E. Tsai, N. Matsuda, H. Iwase, N. Shigyo, L. Sihver and K. Niita, J. Nucl. Sci. Technol. 55, 684 (2018)

[29] M.J. Martin, Nucl. Data Sheets 114, 1497 (2013)

[30] N. Otuka, B. Lalremruata, M.U. Khandaker, A.R. Usman and L.R.M Punte, Radiat. Phys. Chem. 140, 502 (2017)

[31] D.A. Brown et.al., Nucl. Data Sheets 148, 1 (2018)

[32] A.J. Koning et.al., J. Korean Phys. Soc. 59, 1057 (2011)

[33] A.I. Blokhin et.al., Yad. Reak. Konst. No. 2, 62 (2016)

[34] Z.G. Geet.al., J. Korean Phys. Soc. 59, 1052 (2011)

[35] K. Shibata et.al., J. Nucl. Sci. Technol. 48, 1 (2011)

[36] N. Afroze, M.S. Uddin, S.M. Hossain, M.A. Islam, M.A. Shariff, A.K.M. Zakaria, T.K. Datta and S.M. Azharul Islam, Nucl. Instrum. Meth. Phys. Res. B 336, 1 (2014)

[37] M.S. Uddin, M.H. Chowdhury, S.M. Hossain, Sk.A. Latif, M.A. Hafiz, M.A. Islam, A.K.M. Zakaria, S.M. Yunus and S.M. Azharul Islam, Nucl. Instrum. Meth. Phys. Res. B 266, 3341 (2008) 